PROCEEDINGS OF THE

AMERICAN MATHEMATICAL SOCIETY

Volume 136, Number 6, June 2008, Pages 1933-1943

S 0002-9939(08)09182-X

Article electronically published on February 19, 2008

\title{
NORMALIZATION OF MONOMIAL IDEALS AND HILBERT FUNCTIONS
}

\author{
RAFAEL H. VILLARREAL
}

(Communicated by Bernd Ulrich)

\begin{abstract}
We study the normalization of a monomial ideal, and show how to compute its Hilbert function (using Ehrhart polynomials) if the ideal is zero dimensional. A positive lower bound for the second coefficient of the Hilbert polynomial is shown.
\end{abstract}

\section{INTRODUCTION}

Let $R=k\left[x_{1}, \ldots, x_{d}\right]$ be a polynomial ring over a field $k$ and let $I$ be a monomial ideal of $R$ minimally generated by $x^{v_{1}}, \ldots, x^{v_{q}}$. As usual for $a=\left(a_{i}\right)$ in $\mathbb{N}^{d}$ we set $x^{a}=x_{1}^{a_{1}} \cdots x_{d}^{a_{d}}$. If $\mathcal{R}$ is the Rees algebra of $I, \mathcal{R}=R[I t]$, we call its integral closure $\overline{\mathcal{R}}$ the normalization of $I$. This algebra has for components the integral closures of the powers of $I$ :

$$
\mathcal{R}=R \oplus I t \oplus \cdots \oplus I^{i} t^{i} \oplus \cdots \subset R \oplus \bar{I} t \oplus \cdots \oplus \overline{I^{i}} t^{i} \oplus \cdots=\overline{\mathcal{R}} .
$$

In our situation $\mathcal{R} \subset \overline{\mathcal{R}}$ is a finite extension. By a result of Vasconcelos 14, Theorem 7.58], the $I$-filtration $\mathcal{F}=\left\{\overline{I^{i}}\right\}_{i=0}^{\infty}$ stabilizes for $i \geq d$, i.e., $\overline{I^{i}}=I \overline{I^{i-1}}$ for $i \geq d$. We complement this result by showing that if $\operatorname{deg}\left(x^{v_{i}}\right)=r$ for all $i$, then $\mathcal{F}$ stabilizes for $i$ greater than or equal to the minimum of $\operatorname{rank}\left(v_{1}, \ldots, v_{q}\right)$ and $d-\lfloor d / r\rfloor+1$ (Proposition 2.1 and Corollary 2.7).

If $\operatorname{dim}(R / I)=0$, we are interested in studying the Hilbert function of $\mathcal{F}$ :

$$
f(n)=\ell_{R}\left(R / \overline{I^{n}}\right)=c_{d} n^{d}+c_{d-1} n^{d-1}+\cdots+c_{1} n+c_{0} \quad\left(c_{i} \in \mathbb{Q} ; n \gg 0\right) .
$$

We will express $f(n)$ as a difference of two Ehrhart polynomials (Proposition 3.6) and show a lower bound for $c_{d-1}$ (Proposition 3.16). In particular we obtain an efficient way of computing the Hilbert function of $\mathcal{F}$ using integer programming methods. As an application we show that $e_{0}(d-1)-2 e_{1} \geq d-1$, where $e_{i}$ is the $i$ th Hilbert coefficient of $f$. For monomial ideals this improves the inequality $e_{0}(d-1) \geq 2 e_{1}$ given by Polini, Ulrich and Vasconcelos [12, Theorem 3.2] that holds for an arbitrary $\mathfrak{m}$-primary ideal $I$ of a regular local ring $(R, \mathfrak{m})$. This inequality turns out to be useful to bound the length of divisorial chains for classes of Rees algebras [12, Corollary 3.5].

Received by the editors September 15, 2005, and, in revised form, January 7, 2007.

2000 Mathematics Subject Classification. Primary 13B22; Secondary 13D40, 13F20.

Key words and phrases. Normalization, Hilbert polynomial, monomial ideal, Rees algebra.

This work was partially supported by CONACyT grant 49251-F and SNI, México. 
In the sequel we use [4, 13] as references for standard terminology and notation on commutative algebra and polyhedral geometry. We denote the set of non-negative real (resp. integer, rational) numbers by $\mathbb{R}_{+}\left(\operatorname{resp} . \mathbb{N}, \mathbb{Q}_{+}\right)$.

\section{Normalization of MONOMial IDEALS}

To avoid repetition, we continue to use the notation and definitions used in the Introduction.

Proposition 2.1. Let $r_{0}$ be the rank of the matrix $\left(v_{1}, \ldots, v_{q}\right)$. If $v_{1}, \ldots, v_{q}$ lie in a hyperplane of $\mathbb{R}^{d}$ not containing the origin, then $\overline{I^{b}}=I \overline{I^{b-1}}$ for $b \geq r_{0}$.

Proof. Let $\mathbb{Q}_{+} \mathcal{A}^{\prime}$ be the cone in $\mathbb{Q}^{d+1}$ generated by the set

$$
\mathcal{A}^{\prime}=\left\{\left(v_{1}, 1\right), \ldots,\left(v_{q}, 1\right), e_{1}, \ldots, e_{d}\right\}
$$

where $e_{i}$ is the $i$ th unit vector in $\mathbb{Q}^{d+1}$. Assume $b \geq r_{0}$. Notice that we invariably have $I \overline{I^{b-1}} \subset \overline{I^{b}}$. To show the reverse inclusion take $x^{\alpha} \in \overline{I^{b}}$, i.e., $x^{m \alpha} \in I^{b m}$ for some $0 \neq m \in \mathbb{N}$. Hence $(\alpha, b) \in \mathbb{Q}_{+} \mathcal{A}^{\prime}$. Applying Carathéodory's theorem for cones [13, Corollary 7.1i], we can write

$$
(\alpha, b)=\lambda_{1}\left(v_{i_{1}}, 1\right)+\cdots+\lambda_{r}\left(v_{i_{r}}, 1\right)+\mu_{1} e_{j_{1}}+\cdots+\mu_{s} e_{j_{s}} \quad\left(\lambda_{\ell}, \mu_{k} \in \mathbb{Q}_{+}\right),
$$

where $\left\{\left(v_{i_{1}}, 1\right), \ldots,\left(v_{i_{r}}, 1\right), e_{j_{1}}, \ldots, e_{j_{s}}\right\}$ is a linearly independent set contained in $\mathcal{A}^{\prime}$. Notice that $v_{i_{1}}, \ldots, v_{i_{r}}$ are also linearly independent because they lie in a hyperplane not containing the origin. Hence $r \leq r_{0}$. Since $b=\lambda_{1}+\cdots+\lambda_{r}$, we obtain that $\lambda_{\ell} \geq 1$ for some $\ell$, say $\ell=1$. Then

$$
\alpha=v_{i_{1}}+\left(\lambda_{1}-1\right) v_{i_{1}}+\lambda_{2} v_{i_{2}}+\cdots+\lambda_{r} v_{i_{r}}+\mu_{1} e_{j_{1}}+\cdots+\mu_{s} e_{j_{s}}
$$

and consequently $x^{\alpha} \in I \overline{I^{b-1}}$.

Remark 2.2. In this proof we may replace $\mathbb{Q}$ by $\mathbb{R}$ because according to [15, p. 219] we have the equality $\mathbb{Z}^{d+1} \cap \mathbb{R}_{+} \mathcal{A}^{\prime}=\mathbb{Z}^{d+1} \cap \mathbb{Q}_{+} \mathcal{A}^{\prime}$.

Lemma 2.3. Let $\mathcal{A}^{\prime}=\left\{e_{1}, \ldots, e_{d}\right\} \cup\left\{\left(a_{1}, \ldots, a_{d}, 1\right) \mid a_{i} \in \mathbb{N} ; \sum_{i} a_{i}=r\right\}$, where $r \geq 2$ is an integer. Then the irreducible representation of the cone $\mathbb{R}_{+} \mathcal{A}^{\prime}$, as an intersection of closed halfspaces, is given by

$$
\mathbb{R}_{+} \mathcal{A}^{\prime}=H_{e_{1}}^{+} \cap \cdots \cap H_{e_{d}}^{+} \cap H_{e_{d+1}}^{+} \cap H_{a}^{+},
$$

where $a=(1, \ldots, 1,-r)$ and $H_{a}^{+}=\left\{x \in \mathbb{R}^{d+1} \mid\langle x, a\rangle \geq 0\right\}$.

Proof. We set $\mathcal{A}=\left\{e_{1}, \ldots, e_{d}, r e_{1}+e_{d+1}, \ldots, r e_{d}+e_{d+1}\right\}$ and $N=\left\{e_{1}, \ldots, e_{d+1}, a\right\}$. The cone $\mathbb{R}_{+} \mathcal{A}^{\prime}$ has dimension $d+1$ and one has the equality $\mathbb{R}_{+} \mathcal{A}^{\prime}=\mathbb{R}_{+} \mathcal{A}$. Thus it suffices to prove that $F$ is a facet of $\mathbb{R}_{+} \mathcal{A}$ if and only if $F=H_{b} \cap \mathbb{R}_{+} \mathcal{A}$ for some $b \in N$. Let $1 \leq i \leq d+1$. Consider the following sets of vectors:

$$
\Gamma_{i}=\left\{e_{1}, e_{2}, \ldots, \widehat{e}_{i}, \ldots, e_{d}, e_{d+1}\right\} \text { and } \Gamma=\left\{r e_{1}+e_{d+1}, \ldots, r e_{d}+e_{d+1}\right\},
$$

where $\widehat{e}_{i}$ means to omit $e_{i}$ from the list. Since $\Gamma_{i}$ and $\Gamma$ are linearly independent, we obtain that $F=H_{b} \cap \mathbb{R}_{+} \mathcal{A}$ is a facet for $b \in N$, i.e., $\operatorname{dim}(F)=d$ and $\mathbb{R}_{+} \mathcal{A} \subset H_{b}^{+}$. Conversely let $F$ be a facet of the cone $\mathbb{R}_{+} \mathcal{A}$. There are linearly independent vectors $\alpha_{1}, \ldots, \alpha_{d} \in \mathcal{A}$ and $0 \neq b=\left(b_{1}, \ldots, b_{d+1}\right) \in \mathbb{R}^{d+1}$ such that

(i) $F=\mathbb{R}_{+} \mathcal{A} \cap H_{b}$,

(ii) $\mathbb{R} \alpha_{1}+\cdots+\mathbb{R} \alpha_{d}=H_{b}$, and

(iii) $\mathbb{R}_{+} \mathcal{A} \subset H_{b}^{+}$. 
Since $e_{1}, \ldots, e_{d}$ are in $\mathcal{A}$, by (iii) one has

$$
\left\langle e_{i}, b\right\rangle=b_{i} \geq 0 \text { for } i=1, \ldots, d .
$$

Set $\mathcal{B}=\left\{\alpha_{1}, \ldots, \alpha_{d}\right\}$ and consider the matrix $M$ whose rows are the vectors in $\mathcal{B}$. To finish the proof we need only show that there exists $c \in N$ such that $H_{b}=H_{c}$. Consider the following cases. Case (1): If the $i$ th column of $M$ is zero for some $1 \leq i \leq d+1$, we set $c=e_{i}$. Case (2): If $\mathcal{B}=\left\{r e_{1}+e_{d+1}, \ldots, r e_{d}+e_{d+1}\right\}$, then we set $c=a$. Case (3): Now we assume that

$$
\mathcal{B}=\left\{e_{i_{1}}, \ldots, e_{i_{s}}, r e_{j_{1}}+e_{d+1}, \ldots, r e_{j_{t}}+e_{d+1}\right\},
$$

where $s, t>0, s+t=d, 1 \leq i_{1}<\cdots<i_{s} \leq d, 1 \leq j_{1}<\cdots<j_{t} \leq d$, and $M$ has all its columns different from zero. Since $b_{i_{1}}=0$, using

$$
\begin{aligned}
& \left\langle r e_{i_{1}}+e_{d+1}, b\right\rangle=b_{d+1} \geq 0, \\
& \left\langle r e_{j_{1}}+e_{d+1}, b\right\rangle=r b_{j_{1}}+b_{d+1}=0,
\end{aligned}
$$

and equation (2.1), we obtain $b_{d+1}=0$. Then $e_{d+1} \in H_{b}$. It follows readily that $H_{b}$ is generated, as a vector space, by the set $\left\{e_{1}, e_{2}, \ldots, \widehat{e}_{i}, \ldots, e_{d}, e_{d+1}\right\}$ for some $1 \leq i \leq d$. Thus in this case we set $c=e_{i}$.

Let $r \geq 2$ be an integer. For the rest of this section we make two assumptions: (i) $R[t]$ has the grading $\delta$ induced by setting $\delta\left(x_{i}\right)=1$ and $\delta(t)=1-r$, and (ii) $\operatorname{deg}\left(x^{v_{i}}\right)=r$ for all $i$. Thus $\mathcal{R}=R[I t]$ becomes a standard graded $k$-algebra. In this case $\mathcal{R}$ and $\overline{\mathcal{R}}$ have rational Hilbert series. The degree as a rational function of the Hilbert series of $\mathcal{R}$, denoted by $a(\mathcal{R})$, is called the a-invariant of $\mathcal{R}$.

For use below recall that the rth Veronese ideal of $R$ is the ideal generated by all monomials of $R$ of degree $r$. If $x^{a}$ is a monomial we set $\log \left(x^{a}\right)=a$.

Proposition 2.4. Let $J$ be the rth Veronese ideal of $R$ and let $S=R[J t]$ be its Rees algebra. (a) If $r \geq d$, then $a(S)=-2$. (b) If $2 \leq r<d$ and $d=q r+s$, where $0 \leq s<r$, then

$$
a(S)= \begin{cases}-(q+2) & \text { if } \quad s \geq 2, \\ -(q+1) & \text { if } \quad s=0 \text { or } s=1 .\end{cases}
$$

Proof. Let $\mathcal{A}^{\prime}$ be as in Lemma 2.3. As $S$ is normal, according to a formula of Danilov-Stanley [4, the canonical module $\omega_{S}$ of $S$ can be expressed as

$$
\omega_{S}=\left(\left\{x^{a} t^{b} \mid(a, b) \in \mathbb{N} \mathcal{A}^{\prime} \cap\left(\mathbb{R}_{+} \mathcal{A}^{\prime}\right)^{\circ}\right\}\right)=\left(\left\{x^{a} t^{b} \mid(a, b) \in \mathbb{Z}^{d+1} \cap\left(\mathbb{R}_{+} \mathcal{A}^{\prime}\right)^{\circ}\right\}\right),
$$

where $\left(\mathbb{R}_{+} \mathcal{A}^{\prime}\right)^{\circ}$ denotes the relative interior of $\mathbb{R}_{+} \mathcal{A}^{\prime}$ and $\mathbb{N} \mathcal{A}^{\prime}$ is the subsemigroup of $\mathbb{N}^{d+1}$ generated by $\mathcal{A}^{\prime}$. In our situation recall that $a(S)=-\min \left\{i \mid\left(\omega_{S}\right)_{i} \neq 0\right\}$.

Let $m \in \omega_{S}$. We can write $m=x^{a}\left(x^{b} t^{c}\right)$, where $x^{b} t^{c}=\left(f_{1} t\right) \cdots\left(f_{c} t\right)$ and $f_{i}$ is a monomial of degree $r$ for all $i$. Notice that $\delta(m)=|a|+c$, where $a=\left(a_{i}\right)$ and $|a|=a_{1}+\cdots+a_{d}$. Since $\log (m)=(a+b, c)$ is in the interior of the cone $\mathbb{R}_{+} \mathcal{A}^{\prime}$, using Lemma 2.3 one has $c \geq 1, a_{i}+b_{i} \geq 1$ for all $i$, and $|a|+|b| \geq r c+1$. As $|b|=r c$, altogether we get

$$
|a|+|b| \geq d \text { and }|a| \geq 1 .
$$

In particular $\delta(m) \geq 2$. This shows the inequality $a(S) \leq-2$ because $m$ was an arbitrary monomial in $\omega_{S}$. To prove (a) notice that by Lemma 2.3 the monomial $m_{1}=x_{1}^{r-d+2} x_{2} \cdots x_{d} t$ is in $\omega_{S}$ (see the argument below) and $\delta\left(m_{1}\right)=2$. Hence $a(S)=-2$. To prove (b) there are three cases to consider. We only show the case $s \geq 2$; the cases $s=1$ and $s=0$ can be shown similarly. 
Case $s \geq 2$ : First we show that $\delta(m) \geq q+2$. If $c>q$, then from equation (2.3) we get $\delta(m) \geq q+2$. Assume $c \leq q$. One has the inequality

$$
r(q-c)+s \geq(q-c)+2 .
$$

From equation (2.3) one has $|a|+|b|=|a|+r c \geq d=r q+s$. Consequently

$$
\delta(m)=|a|+c \geq r(q-c)+s+c .
$$

Hence from equations (2.4) and (2.5) we get $\delta(m) \geq q+2$. Therefore one has the inequality $a(S) \leq-(q+2)$; to show equality it suffices to prove that the monomial

$$
m_{2}=x_{1}^{2} x_{2}^{2} \cdots x_{r-s+1}^{2} x_{r-s+2} \cdots x_{d} t^{q+1}
$$

is in $\omega_{S}$ and has degree $q+2$. An easy calculation shows that $\delta\left(m_{2}\right)=q+2$. Finally let us see that $m_{2}$ is in $\omega_{S}$ via Lemma 2.3. That the entries of $\log \left(m_{2}\right)$ satisfy $X_{i}>0$ for all $i$ is clear. The inequality

$$
X_{1}+X_{2}+\cdots+X_{d}>r X_{d+1},
$$

after making $X_{i}$ equal to the $i$ th entry of $\log \left(m_{2}\right)$, transforms into

$$
2(r-s+1)+(d-(r-s+1))>r(q+1),
$$

but the left hand side is $r(q+1)+1$, hence $\log \left(m_{2}\right)$ satisfies equation (2.6). Hence $\log \left(m_{2}\right)$ is in the interior of $\mathbb{R}_{+} \mathcal{A}^{\prime}$, i.e., $m_{2} \in \omega_{S}$.

For some other explicit formulae of $a$-invariants see [2]. The next result sharpens 6. Theorem 3.3] for the class of ideals generated by monomials of the same degree.

Proposition 2.5. If $2 \leq r<d$, then the normalization $\overline{\mathcal{R}}$ of $I$ is generated as an $\mathcal{R}$-module by elements $g \in R[t]$ of $t$-degree at most $d-\lfloor d / r\rfloor$.

Proof. Set $f_{i}=x^{v_{i}}$ for $i=1, \ldots, q$. Consider the subsemigroup $C$ of $\mathbb{N}^{d+1}$ generated by the set $\left\{e_{1}, \ldots, e_{d},\left(v_{1}, 1\right), \ldots,\left(v_{q}, 1\right)\right\}$ and the subgroup $\mathbb{Z} C$ generated by $C$. Since $\mathbb{Z} C=\mathbb{Z}^{d+1}$, the normalization of $I$ can be expressed as

$$
\overline{\mathcal{R}}=k\left[\left\{x^{a} t^{b} \mid(a, b) \in \mathbb{Z}^{d+1} \cap \mathbb{R}_{+} C\right\}\right] .
$$

Let $m=x^{a} t^{b}$ be a monomial of $\overline{\mathcal{R}}$ with $(a, b) \neq 0$. We claim that $\delta(m) \geq b$. To show this inequality write

$$
(a, b)=\lambda_{1} e_{1}+\cdots+\lambda_{d} e_{d}+\mu_{1} \log \left(f_{1} t\right)+\cdots+\mu_{q} \log \left(f_{q} t\right),
$$

where $\lambda_{i} \geq 0, \mu_{j} \geq 0$ for all $i, j$. Hence

$$
|a|=\lambda_{1}+\cdots+\lambda_{d}+\left(\mu_{1}+\cdots+\mu_{q}\right) r \quad \text { and } \quad b=\mu_{1}+\cdots+\mu_{q} .
$$

Consequently $\delta(m)=|a|+(1-r) b=\left(\lambda_{1}+\cdots+\lambda_{d}\right)+b \geq b$. We may assume that $k$ is infinite. There is a Noether normalization $A=k\left[z_{1}, \ldots, z_{d+1}\right] \stackrel{\varphi}{\hookrightarrow} \mathcal{R}$ such that $z_{1}, \ldots, z_{d+1} \in \mathcal{R}_{1}$. If $\psi$ is the inclusion from $\mathcal{R}$ to $\overline{\mathcal{R}}$, note that $A \stackrel{\psi \varphi}{\longrightarrow} \overline{\mathcal{R}}$ is a Noether normalization. By [9], the $\operatorname{ring} \overline{\mathcal{R}}$ is Cohen-Macaulay. Hence $\overline{\mathcal{R}}$ is a free $A$-module that according to [15, Proposition 2.2.14] can be written as

$$
\overline{\mathcal{R}}=A m_{1} \oplus \cdots \oplus A m_{n},
$$

where $m_{i}=x^{\beta_{i}} t^{b_{i}}$. Set $h_{i}=\left|\left\{j \mid \delta\left(m_{j}\right)=i\right\}\right|$. Using the fact that the length is additive we obtain the following expression for the Hilbert series of $\overline{\mathcal{R}}$ :

$$
H(\overline{\mathcal{R}}, z)=\sum_{i=0}^{n} \frac{z^{\delta\left(m_{i}\right)}}{(1-z)^{d+1}}=\frac{h_{0}+h_{1} z+\cdots+h_{s} z^{s}}{(1-z)^{d+1}} .
$$


Recall that $a(\overline{\mathcal{R}})=-\min \left\{i \mid\left(\omega_{\overline{\mathcal{R}}}\right)_{i} \neq 0\right\}$, where $\omega_{\overline{\mathcal{R}}}$ is the canonical module of $\overline{\mathcal{R}}$. Let $J$ be the $r$ th Veronese ideal of $R$ and let $S=R[J t]$ be its Rees algebra. Notice that $\overline{\mathcal{R}} \subset S$ because $R[I t] \subset S$ and $S$ is normal. Since $\operatorname{dim}(\overline{\mathcal{R}})=\operatorname{dim}(S)=d+1$, from the Danilov-Stanley formula (see equation (2.2) ) it is seen that $a(\overline{\mathcal{R}}) \leq a(S)$; see the proof of [6, Proposition 3.5]. Therefore using Proposition 2.4 we get

$$
a(\overline{\mathcal{R}})=s-(d+1) \leq a(R[J t]) \leq-\lfloor d / r\rfloor-1,
$$

and $s \leq d-\lfloor d / r\rfloor$. Altogether if $m_{i}=x^{\beta_{i}} t^{b_{i}}$, one has

$$
b_{i} \leq \delta\left(m_{i}\right) \leq s \leq d+1+a(R[J t]) \leq d-\lfloor d / r\rfloor .
$$

Therefore $b_{i}$, the $t$-degree of $m_{i}$, is less than or equal to $d-\lfloor d / r\rfloor$, as required.

Proposition 2.6. $\overline{I^{b}}=I \overline{I^{b-1}}$ for $b \geq d+2+a(R[J t])$.

Proof. It suffices to prove the inclusion $\overline{I^{b}} \subset I \overline{I^{b-1}}$. Let $x^{a} \in \overline{I^{b}}$, i.e., $m=x^{a} t^{b} \in \overline{\mathcal{R}}$. From equation (2.7) and noticing that $A \subset \mathcal{R}$, we can write $m=\left(x^{\gamma} t^{c}\right) m_{i}$ for some $i$, where $m_{i}=x^{\beta_{i}} t^{b_{i}}$ and $x^{\gamma} \in I^{c}$. Using equation (2.8) gives $c \geq 1$. Thus $x^{a} \in I^{c} \overline{I^{b_{i}}}$. To complete the proof notice that $I^{c} \overline{I^{b_{i}}}=I\left(I^{c-1} \overline{I^{b_{i}}}\right) \subset I \overline{I^{b_{i}+c-1}}=$ $I \overline{I^{b-1}}$.

Corollary 2.7. $\overline{I^{b}}=I \overline{I^{b-1}}$ for $b \geq d-\lfloor d / r\rfloor+1$.

Proof. By Proposition 2.4 one has $a(R[J t]) \leq-\lfloor d / r\rfloor-1$. Hence the result follows applying Proposition 2.6.

\section{Zero Dimensional monomial ideals and Hilbert FunCtions}

Let $R=k\left[x_{1}, \ldots, x_{d}\right]$ be a polynomial ring over a field $k$, with $d \geq 2$, and let $I$ be a zero dimensional monomial ideal of $R$ minimally generated by $x^{v_{1}}, \ldots, x^{v_{q}}$. Here we will study the integral closure of the powers of $I$ and its Hilbert function.

We may assume that $v_{i}=a_{i} e_{i}$ for $1 \leq i \leq d$, where $a_{1}, \ldots, a_{d}$ are positive integers and $e_{i}$ is the $i$ th unit vector of $\mathbb{Q}^{d}$. Set $\alpha_{0}=\left(1 / a_{1}, \ldots, 1 / a_{d}\right)$. We may also assume that $\left\{v_{d+1}, \ldots, v_{s}\right\}$ is the set of $v_{i}$ such that $\left\langle v_{i}, \alpha_{0}\right\rangle<1$, and $\left\{v_{s+1}, \ldots, v_{q}\right\}$ is the set of $v_{i}$ such that $i>d$ and $\left\langle v_{i}, \alpha_{0}\right\rangle \geq 1$. Consider the convex polytopes in $\mathbb{Q}^{d}$ :

$$
P:=\operatorname{conv}\left(v_{1}, \ldots, v_{s}\right), \quad S:=\operatorname{conv}\left(0, v_{1}, \ldots, v_{d}\right)=\left\{x \mid x \geq 0 ;\left\langle x, \alpha_{0}\right\rangle \leq 1\right\},
$$

and the rational convex polyhedron $Q:=\mathbb{Q}_{+}^{d}+\operatorname{conv}\left(v_{1}, \ldots, v_{q}\right)$.

Proposition 3.1 ([8, Proposition 1.1]). $\overline{I^{n}}=\left(\left\{x^{a} \mid a \in n Q \cap \mathbb{Z}^{d}\right\}\right)$ for $0 \neq n \in \mathbb{N}$.

Let us give a simpler expression for $Q$. From the equality

$$
\mathbb{Q}_{+}^{d}+\operatorname{conv}\left(v_{1}, \ldots, v_{d}\right)=\left\{x \mid x \geq 0 ;\left\langle x, \alpha_{0}\right\rangle \geq 1\right\},
$$

we get that $v_{i} \in \mathbb{Q}_{+}^{d}+P$ for $i=1, \ldots, q$. Using the finite basis theorem for polyhedra [13. Corollary 7.1b] we have that $\mathbb{Q}_{+}^{d}+P$ is a convex set. Hence $Q \subset \mathbb{Q}_{+}^{d}+P$, and consequently we obtain the equality

$$
Q=\mathbb{Q}_{+}^{d}+P .
$$

Corollary 3.2. If $\left\langle v_{i}, \alpha_{0}\right\rangle \geq 1$ for all $i$, then $\overline{I^{n}}=\overline{\left(x_{1}^{a_{1}}, \ldots, x_{d}^{a_{d}}\right)^{n}}$ for $n \geq 1$.

Proof. It follows at once from Proposition 3.1 and equation (3.1). Notice that in this case $P=\operatorname{conv}\left(v_{1}, \ldots, v_{d}\right)$. 
The Hilbert function of the filtration $\mathcal{F}=\left\{\overline{I^{n}}\right\}_{n=0}^{\infty}$ is defined as

$$
f(n)=\ell\left(R / \overline{I^{n}}\right)=\operatorname{dim}_{k}\left(R / \overline{I^{n}}\right) ; \quad n \in \mathbb{N} \backslash\{0\} ; \quad f(0)=0 .
$$

As usual $\ell\left(R / \overline{I^{n}}\right)$ denotes the length of $R / \overline{I^{n}}$ as an $R$-module. For simplicity we call $f$ the Hilbert function of $\mathcal{F}$.

Corollary 3.3. $f(n)=\ell\left(R / \overline{I^{n}}\right)=\left|\mathbb{N}^{d} \backslash n Q\right|$ for $n \geq 1$.

Proof. The length of $R / \overline{I^{n}}$ equals the dimension of $R / \overline{I^{n}}$ as a $k$-vector space. By Proposition 3.1 the set $\mathcal{B}=\left\{x^{c} \mid c \notin n Q\right\}$ is precisely the set of standard monomials of $R / \overline{I^{n}}$. Thus $\mathcal{B}$ yields a $k$-vector space basis of $R / \overline{I^{n}}$, and the equality follows.

The Hilbert function of $\mathcal{F}$ is a polynomial function of degree $d$ :

$$
f(n)=c_{d} n^{d}+c_{d-1} n^{d-1}+\cdots+c_{1} n+c_{0} \quad(n \gg 0),
$$

where $c_{0}, \ldots, c_{d} \in \mathbb{Q}$ and $c_{d} \neq 0$. The polynomial $c_{d} x^{d}+\cdots+c_{0}$ is called the Hilbert polynomial of $\mathcal{F}$. One has the equality $d ! c_{d}=e(I)=e(\bar{I})$, where $e(I)$ is the multiplicity of $I$; see $[8$. We will express $f(n)$ as a difference of two Ehrhart polynomials and then show a positive lower bound for $c_{d-1}$.

The Ehrhart function of $P$ is the numerical function $\chi_{P}: \mathbb{N} \rightarrow \mathbb{N}$ given by $\chi_{P}(n)=\left|\mathbb{Z}^{d} \cap n P\right|$. This is a polynomial function of degree $d_{1}=\operatorname{dim}(P)$ :

$$
\chi_{P}(n)=b_{d_{1}} n^{d_{1}}+\cdots+b_{1} n+b_{0} \quad(n \gg 0),
$$

where $b_{i} \in \mathbb{Q}$ for all $i$. The polynomial $E_{P}(x)=b_{d_{1}} x^{d_{1}}+\cdots+b_{1} x+b_{0}$ is called the Ehrhart polynomial of $P$. In general some of the coefficients of $E_{P}(x)$ may be negative. It is unknown whether the coefficients are non-negative if the vertices of $P$ have $\{0,1\}$-entries.

Remark 3.4. Some well known properties of $E_{P}$ are (see [4):

(1) $b_{d_{1}}=\operatorname{vol}(P)$, where $\operatorname{vol}(P)$ denotes the relative volume of $P$.

(2) $b_{d_{1}-1}=\left(\sum_{i=1}^{s} \operatorname{vol}\left(F_{i}\right)\right) / 2$, where $F_{1}, \ldots, F_{s}$ are the facets of $P$.

(3) $\chi_{P}(n)=E_{P}(n)$ for all integers $n \geq 0$. In particular $E_{P}(0)=1$.

(4) Reciprocity law of Ehrhart: $E_{P}^{\circ}(n)=(-1)^{d} E_{P}(-n) \quad \forall n \geq 1$, where $E_{P}^{\mathrm{o}}(n)=\left|\mathbb{Z}^{d} \cap(n P)^{\mathrm{o}}\right|$ and $(n P)^{\mathrm{o}}$ is the relative interior of $n P$.

Lemma 3.5. $P=S \cap Q$.

Proof. Clearly $P \subset S \cap Q$. Conversely let $z=\left(z_{i}\right) \in S \cap Q$. Assume that $z \notin P$. By the separating hyperplane theorem [10, Theorem 3.23], there are $0 \neq b=\left(b_{i}\right) \in \mathbb{R}^{d}$ and $c \in \mathbb{R}$ such that $\left\langle b, v_{i}\right\rangle \leq c$ for $i=1, \ldots, s$ and $\langle b, z\rangle>c$. Assume $c>0$. Since $b_{i} a_{i} \leq c$ for all $i$ and $\left\langle\alpha_{0}, z\right\rangle \leq 1$, we get $\langle b, z\rangle \leq\left\langle\alpha_{0}, z\right\rangle c \leq c$, a contradiction. If $c=0$, then $b_{i} \leq 0$ for all $i$ and $\langle b, z\rangle \leq 0$, a contradiction. If $c<0$, we write $z=\delta+p$, for some $\delta \in \mathbb{Q}_{+}^{d}$ and $p \in P$. Then $c<\langle b, z\rangle=\langle b, \delta\rangle+\langle b, p\rangle \leq\langle b, \delta\rangle+c$. Thus $0<\langle b, \delta\rangle$, a contradiction, because $b_{i} \leq 0$ for all $i$.

Proposition 3.6. $f(n)=E_{S}(n)-E_{P}(n)$ for $n \in \mathbb{N}$. In particular

$$
f(n)=c_{d} n^{d}+c_{d-1} n^{d-1}+\cdots+c_{1} n+c_{0} \text { for } n \in \mathbb{N} \text { and } c_{0}=0 .
$$

Proof. Since $E_{P}(0)=E_{S}(0)=1$, we get the equality at $n=0$. Assume $n \geq 1$. Using Lemma 3.5, we get the decomposition $Q=\left(\mathbb{Q}_{+}^{d} \backslash S\right) \cup P$. Hence

$$
n Q=\left(\mathbb{Q}_{+}^{d} \backslash n S\right) \cup n P \Longrightarrow \mathbb{N}^{d} \backslash n Q=\left[\mathbb{N}^{d} \cap(n S)\right] \backslash\left[\mathbb{N}^{d} \cap(n P)\right] .
$$

Therefore by Corollary 3.3 we obtain $f(n)=E_{S}(n)-E_{P}(n)$. 
Example 3.7. Let $I=\left(x_{1}^{4}, x_{2}^{5}, x_{3}^{6}, x_{1} x_{2} x_{3}^{2}\right)$. Notice that

$$
P=\operatorname{conv}((4,0,0),(0,5,0),(0,0,6),(1,1,2)) .
$$

Using Normaliz [5], to compute the Ehrhart polynomials of $S$ and $P$, we get

$$
\begin{aligned}
& f(n)=E_{S}(n)-E_{P}(n)=\left(1+6 n+19 n^{2}+20 n^{3}\right) \\
& \quad-\left(1+(1 / 6) n+(3 / 2) n^{2}+(13 / 3) n^{3}\right)=(35 / 6) n+(35 / 2) n^{2}+(47 / 3) n^{3} .
\end{aligned}
$$

Theorem 3.8 ([14, Theorem 7.58]). $\overline{I^{b}}=I \overline{I^{b-1}}$ for $b \geq d$.

Remark 3.9. We can use polynomial interpolation together with Theorem 3.8 and Proposition 3.6 to determine $c_{1}, \ldots, c_{d}$; see Example 3.10.

Example 3.10. Let $I=\left(x_{1}^{10}, x_{2}^{8}, x_{3}^{5}\right)$. Using $C o C o A$ 7] we obtain that the values of $f$ at $n=0,1,2,3$ are $0,112,704,2176$. By polynomial interpolation we get

$$
f(n)=\ell\left(R / \overline{I^{n}}\right)=(200 / 3) n^{3}+40 x^{2}+(16 / 3) n, \quad \forall n \geq 0 .
$$

Lemma 3.11. Let $\alpha=\left(\alpha_{i}\right)$ and $\beta=\left(\beta_{i}\right)$ be two vectors in $\mathbb{Q}_{+}^{d}$ such that $\alpha_{i}=\beta_{i}$ for $i=1, \ldots, d-1, \beta_{d}>\alpha_{d}$ and $\left\langle\beta, \alpha_{0}\right\rangle<1$. Then

(a) $\beta \in \operatorname{conv}\left(v_{1}, \ldots, v_{d}, \alpha\right)$.

(b) If $\alpha_{i}>0$ for $i=1, \ldots, d-1$, then $\beta \in \operatorname{conv}\left(v_{1}, \ldots, v_{d}, \alpha\right)^{\circ}$.

(c) If $\alpha_{i}>0$ for $i=1, \ldots, d-1$ and $\alpha \in P$, then $\beta \in P^{\circ}$.

Proof. (a) To see that $\beta$ is a convex combination of $v_{1}, \ldots, v_{d}, \alpha$ we set:

$$
\begin{aligned}
s & =\sum_{i=1}^{d} \alpha_{i} / a_{i}=\left\langle\alpha_{0}, \alpha\right\rangle<1, \quad \mu=1-\left[\frac{\beta_{d}-\alpha_{d}}{a_{d}(1-s)}\right]>0, \\
\lambda_{i} & =(1-\mu) \alpha_{i} / a_{i} \geq 0, \quad i=1, \ldots, d-1, \\
\lambda_{d} & =\left(\beta_{d}-\mu \alpha_{d}\right) / a_{d}=\left(\left(\beta_{d}-\alpha_{d}\right) / a_{d}\right)+\alpha_{d}(1-\mu) / a_{d}>0 .
\end{aligned}
$$

Then $\beta=\lambda_{1} v_{1}+\cdots+\lambda_{d} v_{d}+\mu \alpha$ and $\lambda_{1}+\cdots+\lambda_{d}+\mu=1$, as required.

(b) Set $V=\left\{v_{1}, \ldots, v_{d}, \alpha\right\}$ and $\Delta=\operatorname{conv}(V)$. Since $V$ is affinely independent, $\Delta$ is a $d$-simplex. From [3, Theorem 7.3], the facets of $\Delta$ are precisely those sets of the form $\operatorname{conv}(W)$, where $W$ is a subset of $V$ having $d$ points. If $\beta$ is not in the interior of $\Delta$, then $\beta$ must lie in its boundary by (a). Therefore $\beta$ lies in some facet of $\Delta$, which rapidly yields a contradiction.

(c) By part (b) we get $\beta \in \operatorname{conv}\left(v_{1}, \ldots, v_{d}, \alpha\right)^{\circ} \subset P^{\mathrm{o}}$, as required.

Notation. The relative boundary of $P$ will be denoted by $\partial P$.

Lemma 3.12. If $\alpha \in \partial P \backslash \operatorname{conv}\left(v_{1}, \ldots, v_{d}\right)$ and $\alpha_{i}>0$ for $i=1, \ldots, d$, then the vector $\alpha^{\prime}=\left(\alpha_{1}, \ldots, \alpha_{d-1}, 0\right)$ is not in $P$.

Proof. Notice that $\left\langle\alpha, \alpha_{0}\right\rangle<1$. If $\alpha^{\prime} \in P$, then by Lemma 3.11(c) we obtain $\alpha \in P^{\circ}$, a contradiction. Thus $\alpha^{\prime} \notin P$.

For use below we set

$$
\begin{aligned}
K_{i} & =\left\{\left(a_{i}\right) \in S \mid a_{i}=0\right\}=\operatorname{conv}\left(\left\{v_{1}, \ldots, v_{d}, 0\right\} \backslash\left\{v_{i}\right\}\right) ; \quad 1 \leq i \leq d, \\
H & =\operatorname{conv}\left(v_{1}, \ldots, v_{d}\right) ; \quad K=\left(\bigcup_{i=1}^{d} K_{i}\right) \backslash H ; \quad L=\partial P \backslash H, \quad \text { if } H \subsetneq P .
\end{aligned}
$$


Consider the map $\psi: L \rightarrow K$ given by

$$
\psi(\alpha)= \begin{cases}\alpha, & \text { if } \alpha_{i}=0 \text { for some } 1 \leq i \leq d, \\ \left(\alpha_{1}, \ldots, \alpha_{d-1}, 0\right), & \text { if } \alpha_{i}>0 \text { for all } 1 \leq i \leq d\end{cases}
$$

Take $\alpha \in L$. Then $\left\langle\alpha, \alpha_{0}\right\rangle\langle 1$. Since $\partial P \subset P \subset S$ it is seen that $\psi(\alpha) \in K$. Indeed if $\alpha_{i}=0$ for some $i$, then $\psi(\alpha)=\alpha \in K_{i} \backslash H$. If $\alpha_{i}>0$ for all $i$, then $\alpha$ is a convex combination of $v_{1}, \ldots, v_{d}, 0$. Hence $\psi(\alpha)$ is a convex combination of $v_{1}, \ldots, v_{d-1}, 0$ and $\psi(\alpha) \in K_{d} \backslash H$.

Lemma 3.13. $\psi$ is injective.

Proof. Let $\alpha=\left(\alpha_{i}\right), \beta=\left(\beta_{i}\right) \in L$. Assume $\psi(\alpha)=\psi(\beta)$. If $\alpha_{i}=0$ for some $i$ and $\beta_{j}=0$ for some $j$, then clearly $\alpha=\beta$. If $\beta_{i}>0$ for $i=1, \ldots, d$ and $\alpha_{j}=0$ for some $j$, then $\alpha_{d}=0$ and $\alpha_{i}=\beta_{i}$ for $i=1, \ldots, d-1$. By Lemma 3.12 we can readily see that this case cannot occur. If $\alpha_{i} \beta_{i}>0$ for all $i$; then $\alpha=\beta$ by Lemma 3.11(c).

Let us introduce some more notation. We set

$$
\begin{aligned}
\mathcal{A}_{i} & =\left\{v_{j} \mid 1 \leq j \leq s ; x_{i} \notin \operatorname{supp}\left(x^{v_{j}}\right)\right\} ; \\
P_{i} & =\operatorname{conv}\left(\mathcal{A}_{i}\right) ; \quad H_{i}=\operatorname{conv}\left(\left\{v_{1}, \ldots, v_{d}\right\} \backslash\left\{v_{i}\right\}\right) \subset P_{i} \subset K_{i} .
\end{aligned}
$$

Lemma 3.14. $\partial P \cap K_{i}=P_{i}$ for $i=1, \ldots, d$.

Proof. For simplicity of notation assume $i=1$. Let $\alpha=\left(\alpha_{i}\right) \in \partial P \cap K_{1}$, then $\alpha \in P$ and $\alpha_{1}=0$. Since $\alpha$ is a convex combination of $v_{1}, \ldots, v_{s}$ it follows rapidly that $\alpha$ is a convex combination of $\mathcal{A}_{1}$, i.e., $\alpha \in P_{1}$. Conversely let $\alpha \in P_{1}$. Clearly $\alpha \in K_{1} \cap P$ because $\mathcal{A}_{1} \subset K_{1} \cap P$. Assume that $\alpha \notin \partial P$. Then $\alpha \in P^{\circ}$. If $\operatorname{dim}(P)=d-1$, we have that $P=\operatorname{conv}\left(v_{1}, \ldots, v_{d}\right)$ and $P$ is a simplex. Thus by [3, Theorem 7.3], the facets of $P$ are $F_{1}, \ldots, F_{d}$, where $F_{i}=\operatorname{conv}\left(v_{1}, \ldots, v_{i-1}, v_{i+1}, \ldots, v_{d}\right)$. The relative boundary of $P$ is equal to $F_{1} \cup \cdots \cup F_{d}$. Hence $\alpha \notin F_{i}$ for all $i$, and we can write $\alpha=\lambda_{1} v_{1}+\cdots+\lambda_{d} v_{d}$, where $\sum_{i=1}^{d} \lambda_{i}=1$ and $0<\lambda_{i}<1$ for $i=1, \ldots, d$. Thus we get $\alpha_{i}>0$ for $i=1, \ldots, d$, a contradiction. If $\operatorname{dim}(P)=d$, then $\alpha \in P^{\mathrm{o}} \subset S^{\mathrm{o}}$. As in the previous case, but now using the fact that $S$ is a $d$-simplex, we get $\alpha_{i}>0$ for all $i$, a contradiction. Hence $\alpha \in \partial P$.

Lemma 3.15 ([1, p. 38]). Let $A_{1}, \ldots, A_{t}$ be finite subsets of a set $S$; then

$$
\left|\bigcup_{i=1}^{t} A_{i}\right|=\sum_{i=1}^{t}\left|A_{i}\right|-\sum_{i<j}^{t}\left|A_{i} \cap A_{j}\right|+\sum_{i<j<k}^{t}\left|A_{i} \cap A_{j} \cap A_{k}\right| \mp \cdots+(-1)^{t-1}\left|\bigcap_{i=1}^{t} A_{i}\right| .
$$

Proposition 3.16. Let $I_{i}$ be the ideal obtained from $I$ by making $x_{i}=0$ and let $e\left(I_{i}\right)$ be its multiplicity. If $c_{d} x^{d}+c_{d-1} x^{d-1}+\cdots+c_{1} x+c_{0}$ is the Hilbert polynomial of the filtration $\mathcal{F}=\left\{\overline{I^{n}}\right\}_{n=0}^{\infty}$, then

$$
2 c_{d-1} \geq \sum_{i=1}^{d-1} \frac{e\left(I_{i}\right)}{(d-1) !}
$$

Proof. Case (I): $\operatorname{dim}(P)=d$. Let $E_{S}(x)=a_{d} x^{d}+\cdots+a_{1} x+1$ (resp. $E_{P}(x)=$ $b_{d} x^{d}+\cdots+b_{1} x+1$ ) be the Ehrhart polynomial of $S$ (resp. P). By Proposition 3.6, we have the equality $c_{i}=a_{i}-b_{i}$ for all $i$. From the decompositions

$$
P=P^{\mathrm{o}} \cup \partial P, \quad S=S^{\mathrm{o}} \cup \partial S, \quad \partial S=K \cup H, \quad \partial P=L \cup H,
$$


and using the reciprocity law of Ehrhart (Remark 3.4) we get:

$$
\begin{aligned}
f(n) & =E_{S}(n)-E_{P}(n) \\
& =E_{S}^{\mathrm{o}}(n)+\left|\partial(n S) \cap \mathbb{Z}^{d}\right|-\left(E_{P}^{\mathrm{o}}(n)+\left|\partial(n P) \cap \mathbb{Z}^{d}\right|\right) \\
& =(-1)^{d} E_{S}(-n)-(-1)^{d} E_{P}(-n)+\left|n K \cap \mathbb{Z}^{d}\right|-\left|n L \cap \mathbb{Z}^{d}\right|
\end{aligned}
$$

for $0 \neq n \in \mathbb{N}$. Therefore, after simplifying this equality, we obtain:

$$
2\left(c_{d-1} n^{d-1}+c_{d-3} n^{d-3}+\text { terms of lower degree }\right)=\left|n K \cap \mathbb{Z}^{d}\right|-\left|n L \cap \mathbb{Z}^{d}\right|=g(n) .
$$

By the comments just before Lemma 3.13, we have the inclusions

$$
\psi(L) \subset M:=\left[\left(\bigcup_{i=1}^{d-1}\left(\partial P \cap K_{i}\right)\right) \cup K_{d}\right] \backslash H \subset K:=\left(\bigcup_{i=1}^{d} K_{i}\right) \backslash H .
$$

Using Lemma 3.14, we obtain

$$
M=\left(\bigcup_{i=1}^{d-1}\left(P_{i} \backslash H_{i}\right)\right) \cup\left(K_{d} \backslash H_{d}\right) \text { and } K=\bigcup_{i=1}^{d}\left(K_{i} \backslash H_{i}\right) .
$$

Set $h(n)=\left|n K \cap \mathbb{Z}^{d}\right|-\left|n M \cap \mathbb{Z}^{d}\right|$. Since $P_{i} \backslash H_{i} \subset P_{i}, K_{i} \backslash H_{i} \subset K_{i}$ for all $i$ and because $P_{i} \cap P_{j}, K_{i} \cap K_{j}$ are polytopes of dimension at most $d-2$ for $i \neq j$, by the inclusion-exclusion principle (Lemma 3.15) we obtain

$$
\begin{aligned}
& h(n)=\sum_{i=1}^{d}\left|n\left(K_{i} \backslash H_{i}\right) \cap \mathbb{Z}^{d}\right|-\sum_{i=1}^{d-1}\left|n\left(P_{i} \backslash H_{i}\right) \cap \mathbb{Z}^{d}\right| \\
& -\left|n\left(K_{d} \backslash H_{d}\right) \cap \mathbb{Z}^{d}\right|+p(n)=\sum_{i=1}^{d-1}\left(E_{K_{i}}(n)-E_{P_{i}}(n)\right)+p(n) \quad(n \gg 0),
\end{aligned}
$$

where $|p(n)|$ is bounded by a polynomial function $P(n)$ of degree at most $d-2$. In particular $\lim _{n \rightarrow \infty}\left(p(n) / n^{d-1}\right)=0$. By Lemma 3.13, the map $\bar{\psi}: n L \rightarrow n \psi(L)$ given by $\bar{\psi}(n \alpha)=n \psi(\alpha)$ is injective. Hence

$$
\bar{\psi}\left(n L \cap \mathbb{Z}^{d}\right) \subset n \psi(L) \cap \mathbb{Z}^{d} \subset n M \cap \mathbb{Z}^{d} \Rightarrow\left|n L \cap \mathbb{Z}^{d}\right| \leq\left|n M \cap \mathbb{Z}^{d}\right| .
$$

Consequently $g(n)=\left|n K \cap \mathbb{Z}^{d}\right|-\left|n L \cap \mathbb{Z}^{d}\right| \geq h(n)$. Altogether we get

$$
2 c_{d-1}=\lim _{n \rightarrow \infty} \frac{g(n)}{n^{d-1}} \geq \lim _{n \rightarrow \infty} \frac{h(n)}{n^{d-1}}=\lim _{n \rightarrow \infty}\left(\frac{\sum_{i=1}^{d-1}\left(E_{K_{i}}(n)-E_{P_{i}}(n)\right)}{n^{d-1}}+\frac{p(n)}{n^{d-1}}\right) .
$$

Therefore the required inequality follows by observing that the polynomial function $f_{i}(n)=E_{K_{i}}(n)-E_{P_{i}}(n)$ is the Hilbert function of $I_{i}$. Thus $f_{i}(n)$ has degree $d-1$ and its leading coefficient is equal to $e\left(I_{i}\right) /(d-1)$ !.

Case (II): $\operatorname{dim}(P)=d-1$. Let $E_{S}(x)=a_{d} x^{d}+\cdots+a_{1} x+1$ (resp. $E_{P}(x)=$ $b_{d-1} x^{d-1}+\cdots+b_{1} x+1$ ) be the Ehrhart polynomial of $S$ (resp. $P$ ). There is an injective map from $n P$ to $n K_{d}$ induced by $\alpha \mapsto\left(\alpha_{1}, \ldots, \alpha_{d-1}, 0\right)$. Hence

$$
\operatorname{vol}(P)=\lim _{n \rightarrow \infty} \frac{\left|\mathbb{Z}^{d} \cap n P\right|}{n^{d-1}} \leq \lim _{n \rightarrow \infty} \frac{\left|\mathbb{Z}^{d} \cap n K_{d}\right|}{n^{d-1}}=\operatorname{vol}\left(K_{d}\right)
$$


The facets of $S$ are $K_{1}, \ldots, K_{d}$ and $K_{d+1}:=P$. Therefore by Proposition 3.6 and using the formulas for $a_{d-1}$ and $b_{d-1}$ (see Remark 3.4) we conclude that

$$
\begin{aligned}
c_{d-1}=a_{d-1}-b_{d-1} & =\frac{1}{2} \sum_{i=1}^{d+1} \operatorname{vol}\left(K_{i}\right)-\operatorname{vol}(P)=-\frac{1}{2} \operatorname{vol}(P)+\frac{1}{2} \sum_{i=1}^{d} \operatorname{vol}\left(K_{i}\right) \\
& \geq \frac{1}{2} \sum_{i=1}^{d-1} \operatorname{vol}\left(K_{i}\right)=\frac{1}{2} \sum_{i=1}^{d-1} \frac{e\left(I_{i}\right)}{(d-1) !} .
\end{aligned}
$$

Let $e_{0}, e_{1}, \ldots, e_{d}$ be the Hilbert coefficients of $\mathcal{F}$. Recall that we have

$$
f(n)=e_{0}\left(\begin{array}{c}
n+d-1 \\
d
\end{array}\right)-e_{1}\left(\begin{array}{c}
n+d-2 \\
d-1
\end{array}\right)+\cdots+(-1)^{d-1} e_{d-1}\left(\begin{array}{c}
n \\
1
\end{array}\right)+(-1)^{d} e_{d},
$$

where $e_{0}=e(I)$ is the multiplicity of $I$ and $c_{d}=e_{0} / d$ !. Notice that $e_{d}=0$ because $f(0)=0$, and $e_{i} \geq 0$ for all $i$; this follows from [11].

Corollary 3.17. $e_{0}(d-1)-2 e_{1} \geq e\left(I_{1}\right)+\cdots+e\left(I_{d-1}\right) \geq d-1$.

Proof. From the equality $c_{d-1}=\frac{1}{d !}\left[e_{0}\left(\begin{array}{c}d \\ 2\end{array}\right)-d e_{1}\right]$ and using Proposition 3.16 we obtain the desired inequality.

Example 3.18. Let $\mathfrak{m}=\left(x_{1}, \ldots, x_{d}\right)$ and let $I=\mathfrak{m}^{k}$. Then

$$
f(n)=\left(\begin{array}{c}
k n+d-1 \\
d
\end{array}\right)=\frac{k^{d}}{d !} n^{d}+\frac{k^{d-1}}{(d-2) ! 2} n^{d-1}+\text { terms of lower degree, }
$$

$e_{0}=k^{d}, e_{1}=(d-1)\left(k^{d}-k^{d-1}\right) / 2$, and we have equality in Proposition 3.16 .

\section{ACKNOWLEDGMENT}

The author thanks Wolmer Vasconcelos for many stimulating discussions.

\section{REFERENCES}

1. M. Aigner, Combinatorial Theory, Springer, 1997. MR.1434477

2. A. Alcántar, Rees algebras of square-free Veronese ideals and their $a$-invariants, Discrete Math. 302 (2005), 7-21. MR2179232 (2007d:13003)

3. A. Brøndsted, Introduction to Convex Polytopes, Springer-Verlag, 1983. MR683612 (84d:52009)

4. W. Bruns and J. Herzog, Cohen-Macaulay Rings, Revised Edition, Cambridge University Press, 1997. MR.1251956 (95h:13020)

5. W. Bruns and R. Koch, Normaliz, computing normalizations of affine semigroups, 2003. Available from: ftp.mathematik.Uni-Osnabrueck.DE/pub/osm/kommalg/software.

6. W. Bruns, W. V. Vasconcelos and R. H. Villarreal, Degree bounds in monomial subrings, Illinois J. Math. 41 (1997), 341-353. MR1458177 (98e:13013)

7. A. Capani, G. Niesi and L. Robbiano, CoCoA: A system for doing computations in commutative algebra. 1998. Available via anonymous ftp from lancelot.dima.unige.it.

8. D. Delfino, A. Taylor, W. V. Vasconcelos, R. H. Villarreal and N. Weininger, Monomial ideals and the computation of multiplicities, Commutative ring theory and applications (Fez, 2001), pp. 87-106, Lecture Notes in Pure and Appl. Math. 231, Dekker, New York, 2003. MR 2029820 (2005g:13015)

9. M. Hochster, Rings of invariants of tori, Cohen-Macaulay rings generated by monomials, and polytopes, Ann. of Math. 96 (1972), 318-337. MR0304376 (46:3511)

10. B. Korte and J. Vygen, Combinatorial Optimization. Theory and Algorithms, Algorithms and Combinatorics 21, Third Edition, Springer-Verlag, Berlin, 2000. MR1764207 (2001g:90002) 
11. T. Marley, The coefficients of the Hilbert polynomial and the reduction number of an ideal, J. London Math. Soc. 40 (1989), 1-8. MR1028910 (90m:13026)

12. C. Polini, B. Ulrich and W. V. Vasconcelos, Normalization of ideals and Briançon-Skoda numbers, Math. Res. Lett. 12 (2005), nos. 5-6, 827-842. MR2189243 (2006m:13006)

13. A. Schrijver, Theory of Linear and Integer Programming, John Wiley \& Sons, Chichester, 1986. MR874114 (88m:90090)

14. W. V. Vasconcelos, Integral Closure, Springer Monographs in Mathematics, Springer-Verlag, Berlin, 2005. MR2153889 (2006m:13007)

15. R. H. Villarreal, Monomial Algebras, Dekker, New York, 2001. MR.1800904 (2002c:13001)

Departamento de Matemáticas, Centro de Investigación y de Estudios Avanzados del IPN, Apartado Postal 14-740, 07000 México City, D.F., Mexico

E-mail address: vila@math.cinvestav.mx 